\title{
Adult Cardiac-Related Deaths: A Reflection of Epidemiologic Transition of Diseases in the Developing World?
}

\author{
Martins Oluwafemi Thomas, Nicholas A. Awolola, Olugbenga O. Olusoji \\ Department of Anatomic and Molecular Pathology, Cardiothoracic Surgery Unit, \\ Lagos University Teaching Hospital/College of Medicine of University of Lagos, Lagos, Nigeria \\ Email: oluwafemithomas@yahoo.com
}

Received September 26, 2012; revised November 2, 2012; accepted November 13, 2012

\begin{abstract}
Background: Epidemiologic transition of diseases is taking place globally. Therefore, it is necessary that more work is done to unravel the situation in respect to cardiac diseases in the developing world. Aim: The research was conducted to ascertain the cardiac causes of death in adults and by extension to further unravel the epidemiologic transition in relation to cardiac diseases in the developing world. Materials and Methods: This is an autopsy study of adult cardiac related causes of death in the year 2010 at the Lagos University Teaching Hospital in Nigeria, West Africa. It was conducted based in a 700-bedded hospital that has a catchment area of about 15 million people. Patients' data (including bio-data, causes of death, etc.) were extracted from autopsy records for the year 2010. The data were entered and analysed with SPSS software. Results: There were 789 autopsies in the year and 99 of deaths were cardiac-related. There was male preponderance with a mean age of $54.2 \pm 1.568$ years. Hypertensive heart disease was the primary disease in $97.0 \%$ of cases in the studied sample. One 76 year old adult had myocardial infarction. There was no case of adult congenital heart disease. Intracerebral haemorrhage was the commonest cause of death. Discussion and Conclusion: Cardiovascular diseases constitute a growing threat to health among Africans. The developing countries are in stages II and III of Omran epidemiologic transition of diseases. This presents a major challenge to a large proportion of the world population with additional problem of poverty and ignorance. A 4-point agenda is suggested to reduce incidence of cardiovascular diseases (and deaths) in the developing world.
\end{abstract}

Keywords: Cardiac; Deaths; Disease; Transition

\section{Introduction}

Epidemiologic transition which is taking place in every part of the world, among all races, ethnic groups and cultures has resulted in global rise in cardiovascular disorders [1]. From the early 1940s through the 1950s, it was popularly believed throughout the world that cardiac diseases were rare among Nigerians [2]. However, the establishment of a cardiac registry in Ibadan in 1964 revealed all types of cardiac diseases, including those requiring surgical intervention [2].

Living in the tropics does not prevent all the cardiac diseases which are or used to be common in non tropical countries. On the contrary, there are some diseases that are fading in importance in many parts of the world but are still common throughout the tropical countries [3].

In addition, there are cardiac conditions which are virtually limited in occurrence to the tropics and there are other more familiar diseases that are different in natural history or have not yet become as important to tropical epidemiologists as they are elsewhere [3].

For example, coronary artery disease is not a major cause of morbidity and mortality in the tropics unlike in the non tropical clime where lifestyles have changed in the course of industrialisation and urbanization [3].

The spectrum of cardiac diseases is very wide. They are either congenital as in the cyanotic and acyanotic cardiac diseases that are inborn or acquired as in rheumatic heart diseases.

In Ethiopia, the top four cardiac diseases comprise rheumatic heart diseases, hypertensive heart disease, cardiomyopathy and ischemic heart disease in reducing order [1].

The spectrum showed a slight departure from a similar study conducted 5 years earlier in the same setting [4]. Hypertensive heart disease had overtaken cardiomyopathy to become the second commonest in prevalence. Symptomatic rheumatic heart disease affects a mean age of 24 years in Nigeria [5]. Way back in 2004, a study of 
sudden cardiac deaths in Nigeria revealed hypertensive heart disease as the commonest cause of sudden cardiac deaths [6]. These accounted for $83.5 \%$ of cases even when only $30.3 \%$ were previously diagnosed before their sudden death.

In the same study, disease specific prevalence ranked hypertensive heart disease as being the commonest, followed by ischemic heart disease (6.3\%) cardiomyopathies (6.3\%) and $1.3 \%$ each for congenital heart diseases, infective endocarditis and acquired valvular heart disease.

Also, in agreement with above position, Akinkugbe [7] and Osuntokun [8] in 1987 concluded that hypertension is the most common cardiovascular disease among Africans and its complications including congestive cardiac failure and cerebrovascular accidents are among the leading non-communicable causes of death.

The burden of hypertensive heart disease is further exemplified by the report of National Survey in non-communicable diseases in Nigeria in 1997.

In the survey, hypertension prevalence rates in rural and urban Nigeria were $9.8 \%$ and $14.6 \%$ respectively, [9].

The overall pattern of cardiac related causes of death differed slightly for males and females in Nigeria [6]. In the study, hypertensive heart disease accounted for higher proportion of deaths among females (95\% compared to $79.7 \%$ in males), while ischemic heart disease and cardiomyopathy played a comparatively higher role among males. With a mean age of 53.7 years, and the prevalence stated above, the economic implication of cardiac disease burden can be better imagined in a resource-poor nation like Nigeria.

The causes of death as reported at autopsies differ significantly. In the reported sudden cardiac death series [6], 83.3\% of hypertensive heart disease patients died of acute left ventricular failure and $16.7 \%$ of them died of congestive cardiac failure.

This study was conducted to further characterise the major events of cardiac epidemiologic transition as they unfold in this part of the world.

\section{Methodology}

This is an autopsy study of cardiac related causes of death from January to December of 2010. The study centre was Lagos University Teaching Hospital in Lagos metropolis. Lagos University Teaching Hospital is a major referral centre in South West Nigeria. Indeed, it is one of the largest centres in West Africa with a high concentration of experts for cardiac case. It is a major player in cardiac service and it serves a catchment area of well above 15 million population with 700-bed capacity admission facility.

Relevant patients' data were extracted from autopsy records of the hospital. All adult autopsy records for 2010 were scanned through to extract the needed data on all cardiac related deaths. We got their bio-data including names, age, gender and initial diagnosis (primary disease).

We also got the autopsy findings including the final diagnosis of the causes of death. The day and month of death were also recorded. All data were entered using SPSS 17 software and same were analysed to derive the needed information. In particular we descriptively analysed the data looking at age and gender distribution, antemortem diagnosis (primary disease) and postmortem diagnosis of causes of death.

We excluded cases of incomplete data. The information thus extracted was used to draw conclusions about cardiac deaths in this environment.

\section{Cardiac Deaths Results}

\subsection{Age Distribution}

There were 789 autopsies $(n=789)$ within the 12 -month study period. Out of these, 99 deaths $(n=99)$ were cardiac related giving a monthly incidence of 8.2. There were 68 males and 30 males giving a M:F ratio of 2.3:1. The gender of one of the patients was not clearly documented so it was excluded from the study.

The age range was 64 years (Minimum 22 years and Maximum 86 years). The age distribution had a mean of $54.2 \pm 1.568$ years. The median was 54.5years and it was a multimodal distribution. The standard deviation was 15.359 years with a negative skewness (standard error of skewness was 0.246). Majority of deaths (65.3\%) occurred in the 36 - 65 year age range leaving only $9.2 \%$ below the 35 year mark. The distribution was also leptokurtic (standard error of kurtosis $=0.488$ ). The multimodal occurrence of deaths with age is as in Figure 1. Ages 45, 46, 60 and 65 years recorded equal frequencies of 6 deaths each.

\subsection{Primary Diseases and Causes of Death}

Hypertensive cardiovascular disease as primary disease, constituted overwhelming majority of $97.0 \%$ of the studied population with only one case each of mitral stenosis, dilated cardiomyopathy and endocarditis (Table 1). This is graphically depicted as Figure 2. No case of adult congenital heart disease was found. Only one 76-year-old adult had myocardial infarction.

There was one case of polycystic kidney disease in a 36-year-old female. Malignant hypertension was found in a 24-year-old female who suffered biventricular failure. There was a case of glomerulonephritis in a 27-year-old male who also died of biventricular failure. Heart failure was the cause of death in 52 patients constituting 54.2\% of deaths while intra-cranial haemorrhage caused 39 deaths (40.6\%) within the same group. Both patient who had dilated cardiomyopathy and endocarditis died of congestive cardiac failure. 


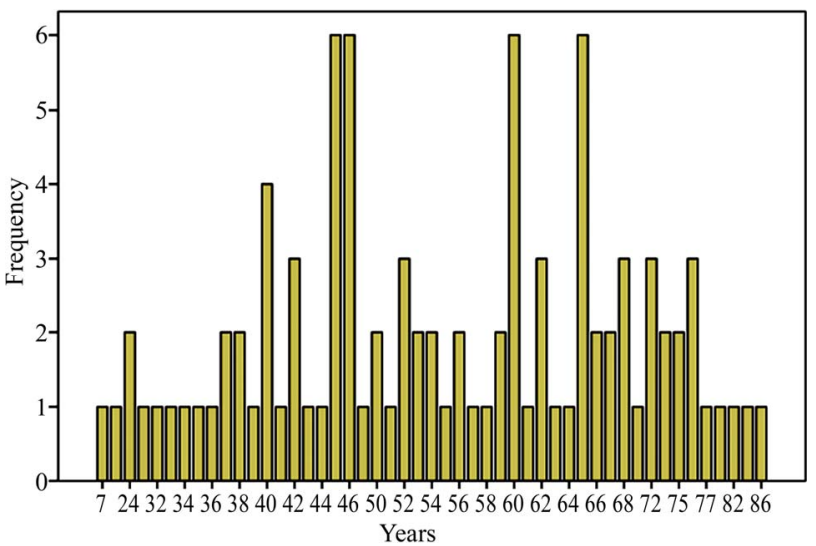

Figure 1. Age distribution of all deaths.

Table 1. Frequency distribution of primary disease.

\begin{tabular}{lcc}
\hline \multicolumn{1}{c}{ Diagnosis } & Frequency & Percentage \\
\hline DCM & 1 & 1.0 \\
ENDOC & 1 & 1.0 \\
HCV & 96 & 97.0 \\
MS & 1 & 1.0 \\
TOTAL & 99 & 100.0 \\
\hline
\end{tabular}

Legend: DCM: Dilated Cardiomyopathy; ENDOC: Endocarditis; HCVD: Hypertensive Cardiovascular Disease; MS: Mitral Stenosis.

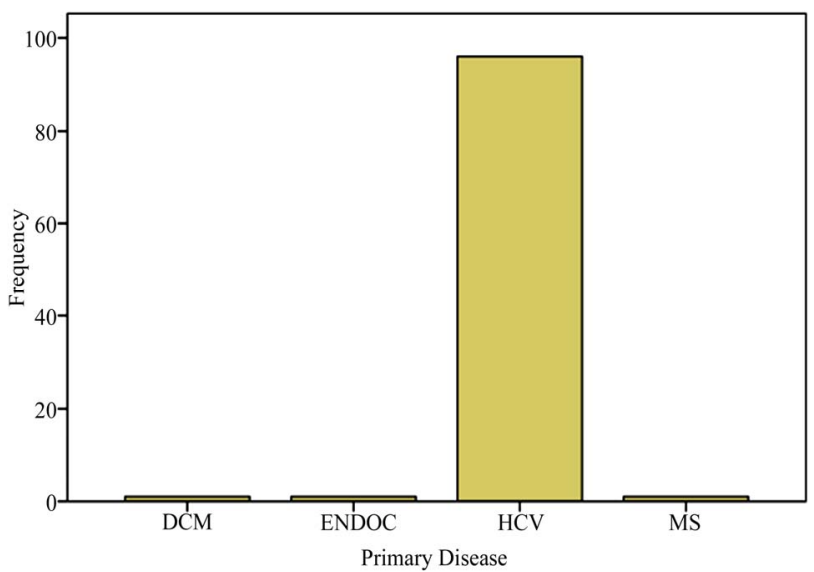

Figure 2. Bar chat of primary diseases.

The causes of death varied widely. Figure $\mathbf{3}$ is the bar chart of the causes of death. The frequency distribution of causes of death is as in Table 2.

The distribution of causes of death was trimodal with intracerebral hemorrhage having the highest frequency of $38.4 \%$, acute left ventricular haemorrhage $31.3 \%$ and biventricular failure having $24.2 \%$ respectively. (Figure 2).

Intracerebral haemorrhage was the commonest cause of death in hypertensive cardiovascular disease (38.0\%). This is closely followed by acute left ventricular failure (31.0\%) and biventricular failure (21.0\%), respectively. (Table 3).

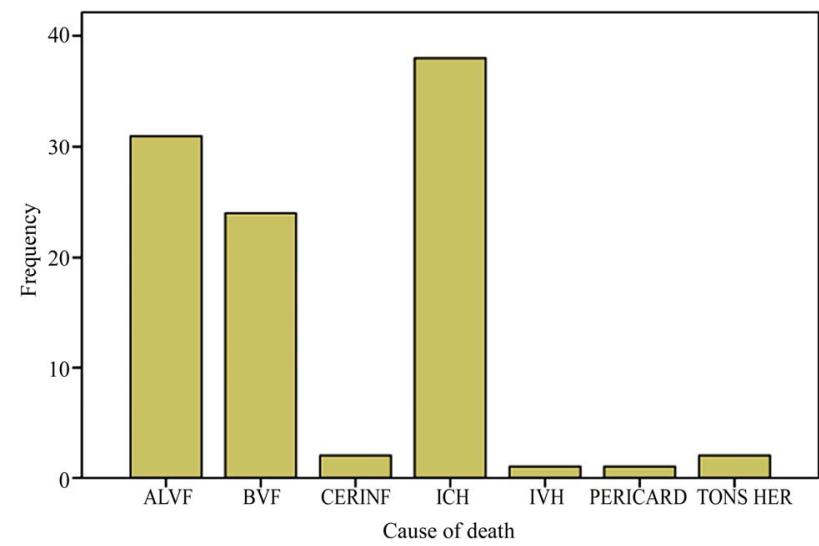

Figure 3. Bar chat of causes of death.

Table 2. Frequency distribution of causes of death.

\begin{tabular}{lcc}
\hline \multicolumn{1}{c}{ Cause of death } & Frequency & Percentage \\
\hline BVF & 24 & 24.2 \\
CER INF & 2 & 2.0 \\
ICH & 38 & 38.4 \\
IVH & 1 & 1.0 \\
PERICARD & 1 & 1.0 \\
TONS HER & 2 & 2.0 \\
Total & 99 & 100.0 \\
\hline
\end{tabular}

Legend: ALVF: Acute Left Ventricular Failure; BVF: Biventricular Failure; CER INF: Cerebral Infarction; ICH: Intracerebral Haemorrhage; IVH: Intraventricular Haemorrahge; PERICARD: Pericarditis; TONS HOR: Tonsilar Herniation.

Table 3. Showing primary diseases and causes of death.

\begin{tabular}{cccccc}
\hline \multicolumn{7}{c}{ Primary Disease } \\
\hline Cause of death & DCM & ENDOC & HCV & MS & Total \\
\hline ALVF & - & - & 31 & - & 31 \\
BVF & 1 & 1 & 21 & 1 & 24 \\
CER INF & - & - & 2 & - & 2 \\
ICH & - & - & 38 & - & 38 \\
IVH & - & - & 1 & - & 1 \\
PERICARD & - & - & 1 & - & 1 \\
TONS HER & - & - & 2 & - & 2 \\
Total & 1 & 1 & 96 & 1 & 99 \\
\hline
\end{tabular}

Legend: ALVF: Acute Left Ventricular Failure; BVF: Biventricular Failure; CER INF: Cerebral Infarction; ICH: Intracerebral Haemorrhage; IVH: Intraventricular Haemorrahage; PERICARD: Pericarditis; TONS HOR: Tonsilar Herniation; DCM: Dilated Cardiomyopathy; ENDOC: Endocarditic; HCV: Hypertensive Cardiovascular Disease; MS: Mitral Stenosis.

A run of Pearson Chi-square test of significance showed a value of 9.668 ( $\mathrm{df}=18$ and asymptomatic significance of 0.942). The likelihood ratio showed a value of 8.802 (df = 18, Asymp. Sig 0.964). Goodman and Krustal directional measured cause of death dependence of 0.037 (standard error $=0.005$ ) and primary diseases dependence of 0.064 (standard error 0.036). 
A check on the dates and frequency of deaths (Figure 5) showed a bimodal distribution in February and November of the year. Therefore, there were more cardiac deaths in February (12 deaths-12.1\%) and November (11 deaths-11.1\%). This is as presented in Figure 4.

\subsection{Relating Sex to Primary Disease and Causes of Deaths}

Hypertensive cardiovascular disease showed a male preponderance of $66.7 \%$ (M:F ratio of $2.3: 1$ ). The Pearson Chi-square was significant with a likelihood ratio of 3.830 (std error 0.280).

Cross tabulation of sex against causes of deaths showed $44.1 \%$ of male deaths due to intracerebral haemorrhage while acute left ventricular haemorrhage and biventricular failure were $32.4 \%$ and $17.6 \%$ respectively.

The commonest cause of death among the females was biventricular failure which occurred in $40.0 \%$ of them (Table 4). It is worthy of note that only one male and one female patient each died of cerebral infarction while intraverticular haemorrhage occurred in only one patienta male. Tonsilar herniation also occurred in one patient. Further analysis of causes of death showed that the mean age for acute left ventricular failure was 60 years (95\% confidence level and 12.1 std deviation). The maximum age was 84 years while the minimum age was 38 years. The distribution was negatively skewed and leptokurtic. The mean age for biventricular failure was 53.4 years ( $P$ $=0.005$, std deviation $=21.9$ ). Lower limit was 7 years and upper limit was 86 years.

The distribution was also negatively skewed and leptokurtic.

Intracerebral haemorrhage as a cause of death had a mean of 49.2 years (95\% confidence level). It had a maximum age of 73 years and minimum of 32 years. Therefore the range (41 years) was very wide. The distribution was positively skewed and it was close to normality because the mean and median were fairly close (49.2 years to 46.0 years).

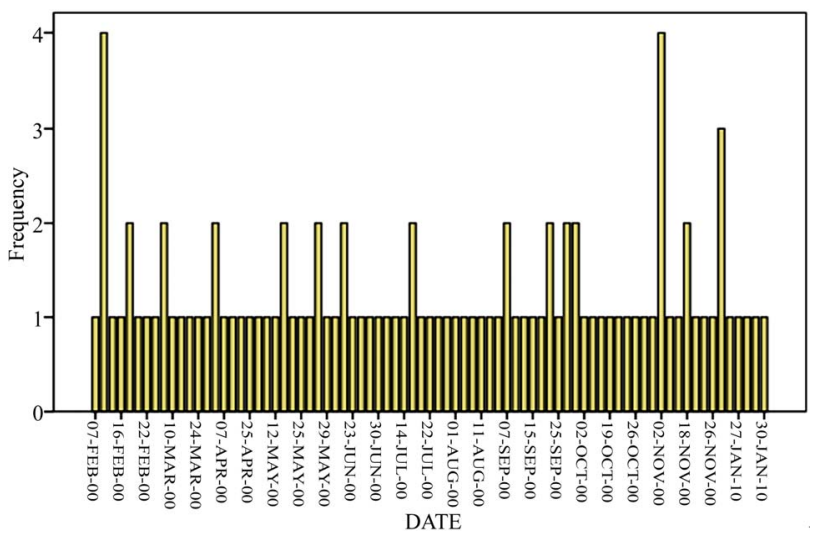

Figure 4. Occurrence of deaths by date.
A stem and leaf plot of age against causes of death (Figure 5) showed biventricular failure with the widest range followed by acute left ventricular failure and intracerebral haemorrhage in that reducing order.

\section{Discussion}

Epidemiology of heart diseases in the tropics has changed tremendously over the last seven decades.

In the 1940s, the situation was such that, cardiac diseases were very rare among Nigerians and by extension, the black Africans.

Over the years, continued change in lifestyle and feeding patterns of Africans has brought major changes in the epidemiologic profile of heart diseases amongst African populations. This has brought non-communicable diseases up as a major burden with cardiac diseases becoming more and more prominent over the years.

Table 4. Gender distribution of causes of death.

\begin{tabular}{ccccccc}
\hline & \multicolumn{5}{c}{ Cause of death } \\
\cline { 2 - 7 } & ALVF & BVF & CER INF & ICH & IVH & PERICARD \\
\hline Male & 22 & 12 & 1 & 30 & 1 & 1 \\
Female & 9 & 12 & 1 & 7 & 0 & 0 \\
Total & 31 & 24 & 2 & 37 & 1 & 1 \\
\hline
\end{tabular}

Legend: ALVF: Acute Left Ventricular Failure; BVF: Biventricular Failure; CER INF: Cerebral Infarction; ICH: Intracerebral Haemorrhage; IVH: Intraventricular Haemorrahge; PERICARD: Pericarditis.

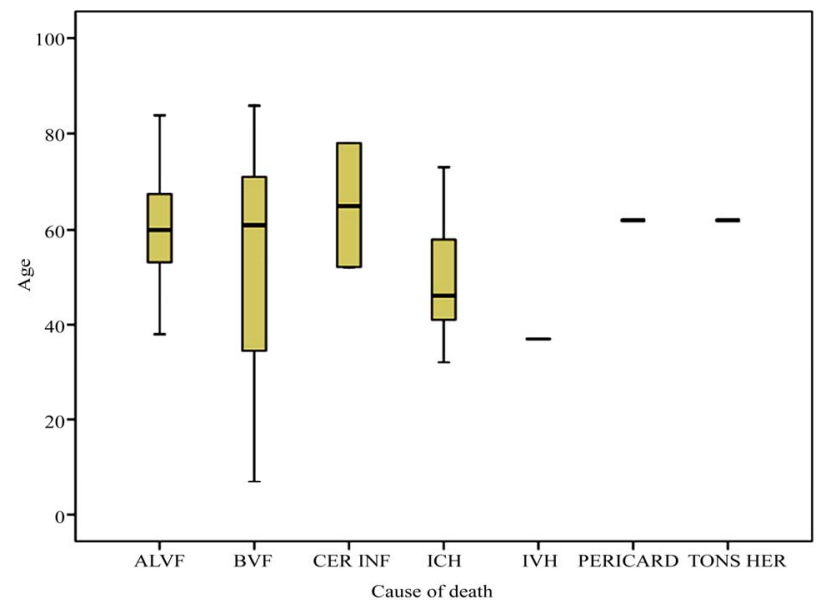

Figure 5. Stem and leaf plot of age against causes of death.

Table 5. Gender distribution of primary diseases.

\begin{tabular}{cccccc}
\hline \multicolumn{7}{c}{ Primary Disease } \\
\hline Gender & DCM & ENDOC & HCV & MS & Total \\
\hline Male & 1 & 1 & 66 & 0 & 68 \\
Female & 0 & 0 & 29 & 1 & 30 \\
Total & 1 & 1 & 95 & 1 & 98 \\
\hline
\end{tabular}

Legend: DCM: Dilated Cardiomyopathy; ENDOC: Endocarditic; HCV: Hypertensive Cardiovascular Disease; MS: Mitral Stenosis. 
Nowadays, literature is awash with increasing profile of cardiac diseases as a major contributor to the burden of non-communicable diseases in Africa. It is therefore apt to continue to study this epidemiologic transition of cardiac disease so that clinicians, epidemiologists and Health Administrators would be abreast of the situation and relevant steps would be taken to address various aspects of the problem as may be necessary.

As a background statement, the demand for postmortem studies in Nigeria is determined by various factors outside the realms of science and law. It is important to know the negative contribution of religion and other social factors to the demand for postmortem in Africa.

These factors perfectly explain the lowering of demand for postmortem as seen in this study. Even at that, 99 cardiac-related deaths (which constitute $12.5 \%$ of all deaths) revealed from autopsy studies over a year period is a major crop in this part of the world.

This further strengthens the description of cardiovascular diseases in an editorial commentary, as a growing threat to health in Africa, accounting for $9.2 \%$ of deaths in 2001 [9]. A gloomy inference from the same publication is the position that cardiovascular diseases have higher mortality rate in developing countries and affect younger people and women disproportionately. In Nigeria, $18.7 \%$ of natural deaths are cardiac related [6].

While we were not able to compare the mortality rates of heart diseases with other diseases in our environment, the study clearly defined a mean age of $54.2 \pm 1$ :568 years and a M:F ratio of 2.3:1 for cardiac deaths. This conforms to other results from South Western Nigeria [6]. The multimodal nature of the distribution caused majority of deaths (65.3\%) to have occurred in the 36 - 65 years age group; thereby substantiating the editorial commentary [9].

Omran model of epidemiology of disease transition divided the transition into 3 stages: pestilence and famine, receding pandemics, and degenerative and man-made diseases [10-19]. Olshansky and Ault [15] added a fourth stage, delayed degenerative diseases. Of particular importance are the 2nd and 3rd stages of disease transition stated above. The 2nd stage (receding pandemics) results in lowering of communicable, maternal, perinatal and nutritional diseases and an increase in cardiovascular risk factors particularly hypertension. The ultimate effect is a greater incidence of cardiovascular diseases, particularly haemorrhagic stroke. The 3rd stage of degenerative and manmade diseases sets the stage for emergence of atherosclerosis.

The worldwide average lifespan has increased beyond 50 years and mortality for cardiovascular diseases has increased with particular preponderance of coronary heart diseases and ischemic stroke. A look at the outcome of our study and other results from Africa [1-6] showed the fact that Africa is indeed enmeshed in the 2nd and 3rd stages of disease epidemic transition presently. Therefore a large chunk of attention needs to be focused on the so-called man-made diseases which are now getting superimposed on the prevailing pandemics of HIV/AIDS and the traditional problem of infant and maternal mortality among others.

This new situation creates a different challenge for the developing world. The prevailing economic stress of such countries will be further worsened if efforts are not doubled to confront the double burden of diseases [16].

The commonest primary disease in our series was hypertensive cardiovascular disease. This constituted an overwhelming majority of $97.0 \%$ of the sample. This conforms with findings of other authors in Nigeria and other countries of Africa $[6,16]$. Aje, had described hypertensive heart disease and failure as the commonest cardiovascular diseases of Africans.

In view of the foregoing and because hypertension is a treatable cardiovascular risk factor, there is need to create more awareness about the disease and educate patients about drug compliance. With these measures, the public health burden of hypertension and its sequelae can be largely reduced.

Necropsy findings of causes of cardiac-related deaths showed that about $70 \%$ of deaths were due to intracranial haemorrhage while about $24.2 \%$ were due to heart failure. This was also the trend in a similar autopsy study in Nigeria where complications of hypertensive heart disease were the causes of death in $83.5 \%$ of cases.

\section{Conclusions}

From the foregoing, we concluded that hypertension control is the key to a reasonably successful drive to reduce cardiac-related deaths in Africa. Any cardiac program that runs short of providing for effective control of hypertension as a front burner issue in Africa stands the risk of failure.

It is in line with this that the Health authorities in Africa, at all levels of care should show more interest in tackling the growing menace of heart diseases (by extension, hypertensive heart disease) in Africa.

We also concluded that the developing countries are in a unique position of epidemiologic transition of diseases. Most of them are in stages II and III of Omran epidemiologic model (stages of receding pandemics, and degenerative and man-made diseases). Cardiovascular diseases and other non-communicable diseases are on the prowl; and the situation is now superimposed on the scourge of poverty, vaccine preventable diseases and other communicable diseases.

A major effect this is weak health system without adequate program for treatment and prevention of cardiovascular diseases. 


\section{REFERENCES}

[1] B. Habte, F. Alemseged and D. Tesfaye, "The Pattern of Cardiac Diseases at the Cardiac Clinic of Jimma University Specialised Hospital, Southwest Ethiopia,” Ethiopian Journal of Health Sciences, Vol. 20, No. 2, 2010, pp. 99105.

[2] J. C. Eze and N. Ezemba, “Open-Heart Surgery in Nigeria,” Texas Heart Institute Journal, Vol. 34, No. 1, 2007, pp. 8-10.

[3] Tropical Medicine Central Resource, An Online Publication, 2006.

http//tmcr.usuhs.mil/tmcr/chapter25/intro.html

[4] G. Petros, "Patterns of Heart Disease Jimma Hospital," Bulletin of Jimma Institute of Health Sciences, Vol. 6, No. 2, 1996, pp. 85-95.

[5] U. Sani, M. Karaye and M. Borado, "Prevalence and Pattern of Rheumatic Heart Disease in the Nigerian Savannah an Echocardiographic Study," Cardiovascular Journal of Africa, Vol. 18, No. 5, 2007, pp. 295-299.

[6] O. Rotimi, A. O. Fatusi and W. O. Odesanmi, "Sudden Cardiac Death in Nigerians. The Ile-Ife Experience," West African Journal of Medicine, Vol. 23, No. 1, 2004, pp. 27-31.

[7] O. O. Akinkugbe, "World Epidemiology of Hypertension in Blacks,” The Journal of Clinical Hypertension, Vol. 3, No. 3, 1987, pp. 15-85.

[8] B. O. Osuntokun, "Stroke in Africans," African Journal of Medicine \& Medical Sciences, Vol. 6, No. 1, 1977, pp. 39-53.
[9] Editorial Commentary, Texas Heart Institute Journal, Vol. 34, No. 1, 2007, pp. 6-7.

[10] T. A. Gaziano, "Cardiovascular Disease in the Developing World and Its Cost-Effective Management," Cardiology Rounds, Vol. 9, No. 2, 2005, pp. 1-6.

[11] C. D. Mathers, A. Lopez, Steinc, D. M. Fat, C. Rao, M. Inoue, K. Shibuya, N. Tomijima, C. Bernard and H. Xu, "Deaths and Disease Burden by Cause: Global Burden of Disease Estimates for 2001, by World Bank Country Groups,” Working Paper 18, Bethesda MD Disease Control Priorities Project.

[12] C. J. Murray and A. D. Lopez, "The Global Burden of Disease: A Comprehensive Assessment of Mortality and Disability from Diseases, Injuries and Risk Factors in 1990 and Projected to 2020," Cambridge Mass, Harvard University, Cambridge, 1996.

[13] A. Rodgers and P. Vanghan, "World Health Report 2002: Reducing Risks, Promoting Healthy Life,” Geneva, 2002.

[14] A. R. Omran, "The Epidemiologic Transition: A Theory of the Epidemiology of Population Change,” Milbank Memorial Fund, Vol. 49, No. 4, 1981, pp. 509-538. doi:10.2307/3349375

[15] S. J. Olshansky and A. B. Ault, "The Fourth Stage of the Epidemiologic Transition: The Age of Delayed Degenerative Diseases," Milbank Memorial Fund, Vol. 64, No. 3, 1986, pp. 355-391.

[16] K. G. Marton, "The Global Impact of Non-Communicable Diseases, Estimates and Projections," World Health Statistics Quarterly, Vol. 41, No. 3-4, 1988, pp. 255-266. 